\section{Raman effect}

The Scientific Papers of C. V. Raman. Vol. 1: The Scattering of Light. Pp. 699. (Indian Academy of Sciences: P.O. Box 621, Bangalore 560006,1978 .) $£ 8$

THE Raman effect is one of the most significant discoveries ever made in spectroscopy. Professor Sir C. V. Raman (1880-1970) was awarded the Nobel prize for physics in 1930 for his work on the scattering of light which resulted in this discovery.

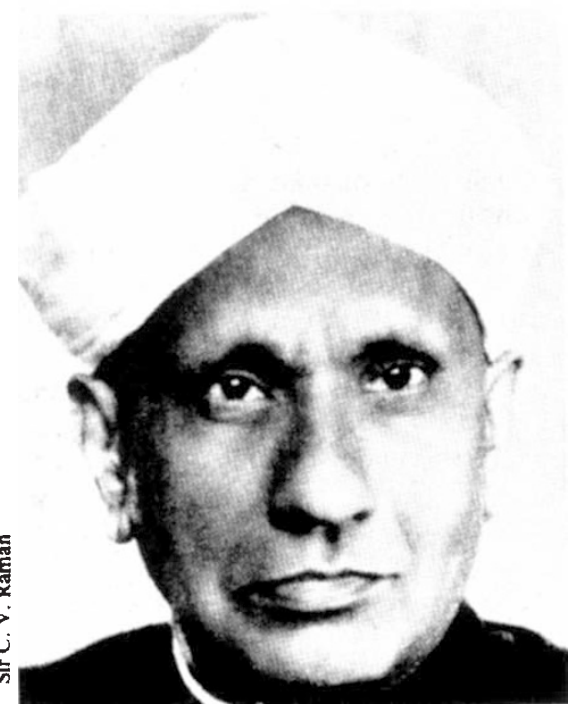

This clearly produced volume contains a selection of 94 of the scientific papers published by Professor Raman, either by himself or along with his students, on the subject of light scattering. It contains an historical introduction to the life and work of this "grand old man" of Indian Science by S. Ramaseshan who was responsible for selecting and editing the papers on behalf of the Indian Academy of Sciences. The collection contains papers on colloid scattering, molecular scattering, surface scattering and Brillouin scattering and also his pioneering publications on molecular anisotropy.

The monograph he wrote in 1922 on the Molecular Diffraction of Light and also his investigations on X-ray scattering and Compton scattering have been included, as these played a significant role in his discovery of the Raman effect in 1928. The intense interest he developed in the scattering of light was due to the visual impact the blue of the Mediterranean sea made on him on his first voyage to Europe in 1921. Even on this journey he was well prepared for experimental observations, for he carried in his pocket Nicol prisms, a small telescope to which polarisers and analysers could be attached, a slit, and even a diffraction grating. In later years he studied the scattering from pure organic materials which S.. Venkateswaran, a parttime worker in his laboratory, had succeeded in purifying by slow distillation in vacuo. Using filtered sunlight to irradiate these liquids in his work with K.S.
Krishnan, Raman observed that all pure organic liquid showed this "feeble fluorescence" and he became convinced that this was the modified scattering of altered wavelength corresponding to the "milder fluctuations" in the state of the scattering molecule. Pointing a direct vision spectroscope on to the scattered track he saw that the scattered light contained not only the incident colour but at least another separated in wavelength from it by a dark space. Then replacing the filtered sunlight by a mercury arc he recorded sharp modified Raman lines for which the frequency shifts were identified with some of the characteristic infrared active vibrational frequencies of the molecule.

He was a man of wide interests in physics and published many papers on sound, ultrasonics, the solid and liquid state as well as optical phenomena. His physical intuitive abilities enabled him to jump several steps in mathematics and his capacity to expound complicated concepts in physics in a simple and appealing manner always made him a popular speaker. The lecture in which he announced his discovery of the Raman

\section{Marine productivity}

Marine Production Mechanisms. International Biological Programme, Vol. 20. (Edited by M. J. Dunbar. Pp. 338. (Cambridge University Press: Cambridge, 1979.) $£ 25$.

LONG before the International Biological Programme (IBP) was thought of, international collaboration was a feature of marine science and it is probably because of this that this synthesis volume from the marine productivity section does not survey the whole field but presents a number of largely unrelated chapters summarising work carried out specifically under IBP auspices. The contributions fall into two groups, regional studies of productivity and ecosystem structure, with an entr'acte in the form of a short chapter by Velasquez on seaweed utilisation in the Phillipines.

The regional studies span seas from the sub-Arctic - primary production in Frobisher Bay, Arctic Canada, is described by Grainger - to the tropics - primary productivity in Indian waters being reviewed by Qasim. Detailed studies of food webs in the coastal waters of Japan are described by Hogetsu, the vertical distribution of phytoplankton in the open Pacific and Atlantic oceans by Semina, and production cycles in the Dutch Wadden Sea by van der Eijk. Parsons touches on mathematical modelling in his account of the Strait of Georgia, British Columbia, but there is no extensive discussion of the potentialities of this approach. Grindley discusses productivity and upwelling in the Benguela region and Dunbar relates
Effect and his Nobel lecture are included in the volume.

Sir C. V. Raman lived long enough to see the whole field of Raman spectroscopy rejuvenated and increased in importance by the use of lasers, which form ideal sources for the observation of the effect. This has enabled much of the earlier work to be repeated under conditions of greater resolution and signal to noise ratio. However, this volume reminds us of the way that Raman blazed new trails by discovering new phenomena such as shear waves in liquids and the 'soft mode' process in crystals. This book will be of value to all interested in light scattering and the Raman effect. At $£ 8$ it represents outstanding value for money as it is a bound volume of nearly 700 pages. Along with the International Raman conference held last year in Bangalore this record of his work forms a fine tribute to the achievements of this outstanding Indian scientist.

\section{G. R. Wilkinson}

G. R. Wilkinson is Professor of Physics at King's College, University of London, UK.

production in the Gulf of St Lawrence to the pattern of water circulation, but the currently fashionable topic of the relationship of production to fronts in the sea has emerged since the IBP was concluded.

The contributions on ecosystem structure are of particular interest in being all by Russian workers. Pepita gives a quantitative account of energy flow in food webs in southern seas of the USSR and the tropical Pacific. A major role in energy supply is attributed to bacterial utilization of dissolved organic matter in tropical waters both by Pepita and by Shushkina and Vinogradov in the following chapter; the contributors to the earlier chapters largely assume that the classical radiocarbon technique of Steemann Nielsen gives an adequate measure of primary production. Neyman and Yablonskaya in the two next chapters employ the useful concept of trophic groups in studies of benthic faunas, and finally Golikov and Scarlato describe the pattern of distribution of benthic faunas in the shelf seas of the USSR. All this is most interesting but few details of methods are given so that, the references being almost exclusively to papers in Russian journals, assessment of the results will be difficult for most of us.

This book will be of great value to the specialist but cannot be recommended as an introduction to the study of marine productivity.

G. E. Fogg

G. E. Fogg is Head of the Department of Marine Biology at the University College of North Wales, Bangor. 